\title{
Infrared/optical - X-ray simultaneous observations of X-ray flares in GRB 071112C and GRB 080506
}

\author{
T. Uehara ${ }^{1}$, M. Uemura ${ }^{2}$, K. S. Kawabata ${ }^{2}$, Y. Fukazawa ${ }^{1}$, R. Yamazaki ${ }^{3}$, A. Arai ${ }^{4}$, M. Sasada ${ }^{1}$, T. Ohsugi ${ }^{2}$,

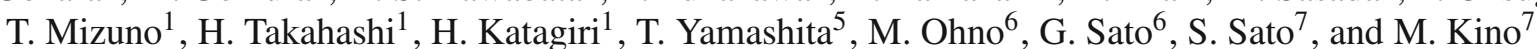 \\ ${ }^{1}$ Department of Physical Science, Hiroshima University, Kagamiyama 1-3-1, Higashi-Hiroshima 739-8526, Japan \\ e-mail: uehara@hep01.hepl.hiroshima-u.ac.jp \\ 2 Hiroshima Astrophysical Science Center, Hiroshima University, Kagamiyama 1-3-1, Higashi-Hiroshima 739-8526, Japan \\ 3 Department of Physics and Mathematics, Aoyama Gakuin University, 5-10-1 Fuchinobe, Sagamihara 252-5258, Japan \\ ${ }^{4}$ Faculty of Science, Kyoto Sangyo University, Motoyama, Kamigamo, Kita-Ku, Kyoto-City 603-8555, Japan \\ 5 National Astronomical Observatory of Japan, 2-21-1 Osawa, Mitaka, Tokyo 181-0015, Japan \\ ${ }^{6}$ Institute of Space and Astronautical Science, JAXA, 3-1-1 Yoshinodai, Sagamihara, Kanagawa 229-8510, Japan \\ 7 Department of Physics, Nagoya University, Furo-cho, Chikusa-ku, Nagoya 464-8602, Japan
}

Received 17 February 2010 / Accepted 25 May 2010

\section{ABSTRACT}

\begin{abstract}
Aims. We investigate the origin of short X-ray flares which are occasionally observed in early stages of afterglows of gamma-ray bursts (GRBs).

Methods. We observed two Swift events, GRB 071112C and GRB 080506, before the start of X-ray flares in the optical and nearinfrared (NIR) bands with the 1.5-m Kanata telescope. In conjunction with published X-ray and optical data, we analyzed densely sampled light curves of the early afterglows and spectral energy distributions (SEDs) in the NIR-X-ray ranges.

Results. We found that the SEDs had a break between the optical and X-ray bands in the normal decay phases of both GRBs regardless of the model for the correction of the interstellar extinction in host galaxies of GRBs. In the X-ray flares, X-ray flux increased by 3 and 15 times in the case of GRB 071112C and 080506, respectively, and the X-ray spectra became harder than those in the normal decay phases. No significant variation in the optical-NIR range was detected together with the X-ray flares.

Conclusions. These results suggest that the X-ray flares were associated with either late internal shocks or external shocks from two-component jets.
\end{abstract}

Key words. gamma-ray burst: individual: GRB 071112C - gamma-ray burst: individual: GRB 080506

\section{Introduction}

Gamma-ray bursts (GRBs) are transient gamma-ray sources whose durations are $\sim 0.1-100 \mathrm{~s}$ (Paciesas et al. 1999). Afterglows are occasionally observed after GRBs in a wide range of wavelengths from radio to X-rays (Costa et al. 1997; van Paradijs et al. 1997; Frail et al. 1997). The most plausible scenario for the origin of GRBs is the internal shock model, in which the gamma-ray emission arises from a shock region generated by collisions between shells in a relativistic jet (Piran 1999; Zhang \& Mészáros 2004). Afterglows are believed to be synchrotron emission from an external shock region between the shell and interstellar medium (Wang \& Loeb 2000; Sari et al. 1998). It is widely acknowledged that a GRB having a long duration of $\sim 2.0-100 \mathrm{~s}$, so-called "long GRBs", is related to a collapse of massive stars based on the fact that its direct connection with a type-Ic supernova has been confirmed (Soderberg et al. 2005; Della Valle et al. 2003; Hjorth et al. 2003; Stanek et al. 2003; Malesani et al. 2004).

X-ray observations with the Swift satellite have discovered short flares in X-ray afterglows $10^{2-4} \mathrm{~s}$ after GRBs. These X-ray flares are unexpected in the framework of the standard external shock model because it predicts a monotonous decay of the X-ray afterglow with a power-law form (Burrows et al. 2005). The X-ray flare is observed in a half of GRB afterglows
(Falcone et al. 2007). The afterglow emission from the external shock could exhibit short-term modulations when a shell passes a high-density region of the interstellar medium (Wang \& Loeb 2000), or slow shells catch up with the main shell (Rees \& Meszaros 1998). Such modulations of the emission from the external shock are candidates for the origin of the X-ray flare. It is also possible that a late-time internal shock causes the X-ray flare (e.g. Burrows et al. 2005; Zhang et al. 2006a; Chincarini et al. 2007; Butler \& Kocevski 2007; Chincarini et al. 2010). This scenario with the late internal shock requires a long activity of the central engine of GRBs (Ioka et al. 2005).

The optical and infrared observations of afterglows are important to evaluate the models for the X-ray flare because the external shock model predicts that an optical-infrared flare is associated with an X-ray flare. No optical-infrared flare has been reported during the $\mathrm{X}$-ray flares in previous observations, while a part of those observations were too sparse to investigate the detailed behavior of optical-infrared afterglows during the X-ray flares (Stanek et al. 2007; Krimm et al. 2007).

Little is known about the temporal variation of spectral energy distributions (SEDs) associated with the X-ray flare. This is because simultaneous multi-wavelength observations are required with a high time-resolution. In addition, it is problematic to correct the interstellar extinction in host galaxies of GRBs 
which is highly uncertain even if such a densely-sampled multiwavelength data is available (e.g. see Stratta et al. 2004; Kann et al. 2006; Chen et al. 2006; Schady et al. 2007; Starling et al. 2007; Watson et al. 2007).

GRB $071112 \mathrm{C}$ and 080506 were detected by Swift/BAT at 18:32:57.54 UT 12 November 2007 (Perri et al. 2007) and 17:46:21.22 UT 6 May 2008 (Baumgartner et al. 2008), respectively. X-ray flares were detected in both GRBs (Stratta et al. 2007; Sbarufatti et al. 2008). Here we report on our optical and NIR observations of those two GRBs using the Kanata 1.5-m telescope. Combined with X-ray spectra, these simultaneous optical and NIR data allowed us to investigate the interstellar extinction in the host galaxies, and thereby, variations of SEDs. We describe the details of our observations in Sect. 2. We report on the temporal evolution of the optical and X-ray afterglows and SEDs in Sect. 3. In Sect. 4, we discuss the origin of the X-ray flare using the internal and external shock models. Finally, we summarize our results in Sect. 5.

\subsection{Optical and NIR observation}

Our observations of GRB 071112C and GRB 080506 started at 18:36:21 (UT) 12 November 2007 and 17:49:51 (UT) 6 May 2008, which were $\sim 324 \mathrm{~s}$ and $\sim 210 \mathrm{~s}$ after the GRB trigger times, respectively. Both observations were performed with TRISPEC attached to the Kanata 1.5-m telescope at HigashiHiroshima Observatory of Hiroshima University. TRISPEC is a simultaneous imager and spectrograph with polarimetry covering both optical and NIR wavelengths (Watanabe et al. 2005). We used the imaging mode of TRISPEC with the $V, J$, and $K_{\mathrm{s}}$ band filters for GRB $071112 \mathrm{C}$. Instead of the $V$-band filter, we used the $R_{\mathrm{C}}$-band filter for GRB 080506 . The observations continued for 4.5 and $5.7 \mathrm{ks}$, and we obtained 40 and 35 sets of three photometric band images for GRB 071112C and GRB 080506, respectively. The central wavelength of the TRISPEC's $R_{\mathrm{C}}$ system is $\sim 620 \mathrm{~nm}$, slightly shifted from the standard one $(=645 \mathrm{~nm})$. The difference of the photometric systems is so small that we neglect it in our following discussion about spectral energy distributions.

We obtained differential magnitudes of the afterglows using a Java-based PSF photometry package after making darksubtracted and flat-fielded images. We used a nearby field-star located at RA $=2^{\mathrm{h}} 36^{\mathrm{m}} 41^{\mathrm{s}} .46$, Dec $=28^{\circ} 20^{\prime} 53^{\prime \prime} .4$ as a comparison star for GRB $071112 \mathrm{C}$. The $V, J$, and $K_{\mathrm{s}}$-band magnitudes of the comparison star were quoted from the Guide Star Catalog Version 2.3.2 $(V=14.43)$ and 2MASS All-Sky Catalog of Point Sources $\left(J=13.238\right.$ and $\left.K_{\mathrm{s}}=12.792\right)$, respectively. We checked systematic errors of $V, J$, and $K_{\mathrm{s}}$ magnitudes depending on comparison stars, and found that it is smaller than $0.28,0.02$, and $0.02 \mathrm{mag}$, using neighboring stars. Uemura et al. (2007b) contains differential magnitudes obtained by our optical observations of GRB $071112 \mathrm{C}$.

For the differential photometry of GRB 080506, we used averages of magnitudes of USNO B1.0 1289-0511223, 12890511261, 1289-0511235, 1289-0511157, and 1289-0511139 for the $R_{\mathrm{C}}$ band, 2MASS 1289-0511197, 1289-0511261, 1289$0511235,1289-0511157$, and $1289-0511139$ for the $J$ band, and 2MASS 1289-0511197 and 1289-0511261 for the $K_{\mathrm{S}}$ band. We checked systematic errors of $R_{\mathrm{C}}, J$, and $K_{\mathrm{s}}$ magnitudes depending on comparison stars, and found that it is smaller than 0.13 , 0.01 , and $0.06 \mathrm{mag}$, using neighboring USNO B1.0 and 2MASS stars. Table 1 contains magnitudes obtained by our observations
Table 1. Kanata optical photometry of GRB 080506.

\begin{tabular}{ccccc}
\hline \hline Filter & Time (s) & Exposure (s) & Mag & Error \\
\hline$R_{\mathrm{C}}$ & 210.0 & 420.0 & 17.48 & \pm 0.22 \\
$R_{\mathrm{C}}$ & 264.0 & 528.0 & 17.59 & \pm 0.27 \\
$R_{\mathrm{C}}$ & 318.0 & 636.0 & 17.50 & \pm 0.24 \\
$R_{\mathrm{C}}$ & 375.0 & 750.0 & 17.81 & \pm 0.33 \\
$R_{\mathrm{C}}$ & 432.0 & 864.0 & 17.72 & \pm 0.26 \\
$R_{\mathrm{C}}$ & 496.0 & 992.0 & 17.82 & \pm 0.28 \\
$R_{\mathrm{C}}$ & 550.0 & 1100.0 & 17.90 & \pm 0.36 \\
$R_{\mathrm{C}}$ & 610.0 & 1220.0 & 17.73 & \pm 0.26 \\
$R_{\mathrm{C}}$ & 665.0 & 1330.0 & 17.89 & \pm 0.30 \\
$R_{\mathrm{C}}$ & 718.0 & 1436.0 & 18.24 & \pm 0.39 \\
$R_{\mathrm{C}}$ & 988.5 & 1977.0 & 18.14 & \pm 0.13 \\
$R_{\mathrm{C}}$ & 1138.5 & 2277.0 & 18.03 & \pm 0.10 \\
$R_{\mathrm{C}}$ & 1288.5 & 2577.0 & 17.98 & \pm 0.10 \\
$R_{\mathrm{C}}$ & 1439.5 & 2879.0 & 18.24 & \pm 0.10 \\
$R_{\mathrm{C}}$ & 1589.5 & 3179.0 & 18.14 & \pm 0.10 \\
$R_{\mathrm{C}}$ & 2820.5 & 5641.0 & 18.96 & \pm 0.30 \\
$R_{\mathrm{C}}$ & 2976.5 & 5953.0 & 18.69 & \pm 0.24 \\
$R_{\mathrm{C}}$ & 3134.5 & 6269.0 & 18.79 & \pm 0.28 \\
$R_{\mathrm{C}}$ & 3288.5 & 6577.0 & 18.88 & \pm 0.33 \\
$R_{\mathrm{C}}$ & 3519.0 & 7038.0 & 18.99 & \pm 0.29 \\
$R_{\mathrm{C}}$ & 3825.0 & 7650.0 & 19.27 & \pm 0.35 \\
$R_{\mathrm{C}}$ & 4136.0 & 8272.0 & 19.11 & \pm 0.28 \\
$R_{\mathrm{C}}$ & 4449.5 & 8899.0 & 19.22 & \pm 0.37 \\
$R_{\mathrm{C}}$ & 4830.2 & 9660.3 & 19.19 & \pm 0.26 \\
$R_{\mathrm{C}}$ & 5371.2 & 10742.3 & 19.31 & \pm 0.37 \\
$J$ & 319.8 & 639.6 & 16.30 & \pm 0.08 \\
$J$ & 607.8 & 1215.6 & 16.64 & \pm 0.13 \\
$J$ & 1063.5 & 2127.0 & 16.40 & \pm 0.05 \\
$J$ & 1439.2 & 2878.3 & 16.62 & \pm 0.06 \\
$J$ & 3132.3 & 6264.6 & 17.71 & \pm 0.27 \\
$J$ & 3903.7 & 7807.4 & 18.14 & \pm 0.39 \\
$J$ & 4677.9 & 9355.8 & 17.76 & \pm 0.38 \\
$K_{\mathrm{s}}$ & 463.8 & 927.6 & 14.71 & \pm 0.09 \\
$K_{\mathrm{s}}$ & 1288.9 & 2577.8 & 15.32 & \pm 0.20 \\
$K_{\mathrm{s}}$ & 3904.6 & 7809.3 & 16.54 & \pm 0.50 \\
\hline${ }$ & & & &
\end{tabular}

Notes. ${ }^{(*)}$ Time since the GRB trigger.

of GRB 080506. In this table, the magnitudes are averages in equally spaced bins in the logarithmic scale of time. The errors include both statistical and systematic ones.

\subsection{XRT analysis of GRB $071112 C$ and GRB 080506}

XRT began observing GRB 071112C and GRB 080506 at 2007 November 12 18:34:27 UT, i.e. at $T+90 \mathrm{~s}$ and 2008 May 6 17:48:47 UT, i.e. at $T+146 \mathrm{~s}$, respectively ( $T$ represents a GRB trigger time). Both XRT data were processed using the HEASOFT package. We extracted both source data with a rectangular $40^{\prime \prime} \times 170^{\prime \prime}$ region for the Windowed Timing mode (WT), and 40" radius region for the Photon Counting mode (PC) from the processed data. Both GRB backgrounds were also extracted from $40^{\prime \prime} \times 170^{\prime \prime}$ source of both ends region for the WT, and 192" internal and 231" outer annulus radius region for the PC, far from the source. Light curves were binned with a requirement of a minimum of 30 photons per bin of WT and 20 photons per bin of PC for GRB 071112C, while 30 photons per bin of WT and 40 photons per bin of PC for GRB 080506. 
T. Uehara et al.: Infrared/optical - X-ray simultaneous observations of X-ray flares in GRB 071112C and GRB 080506
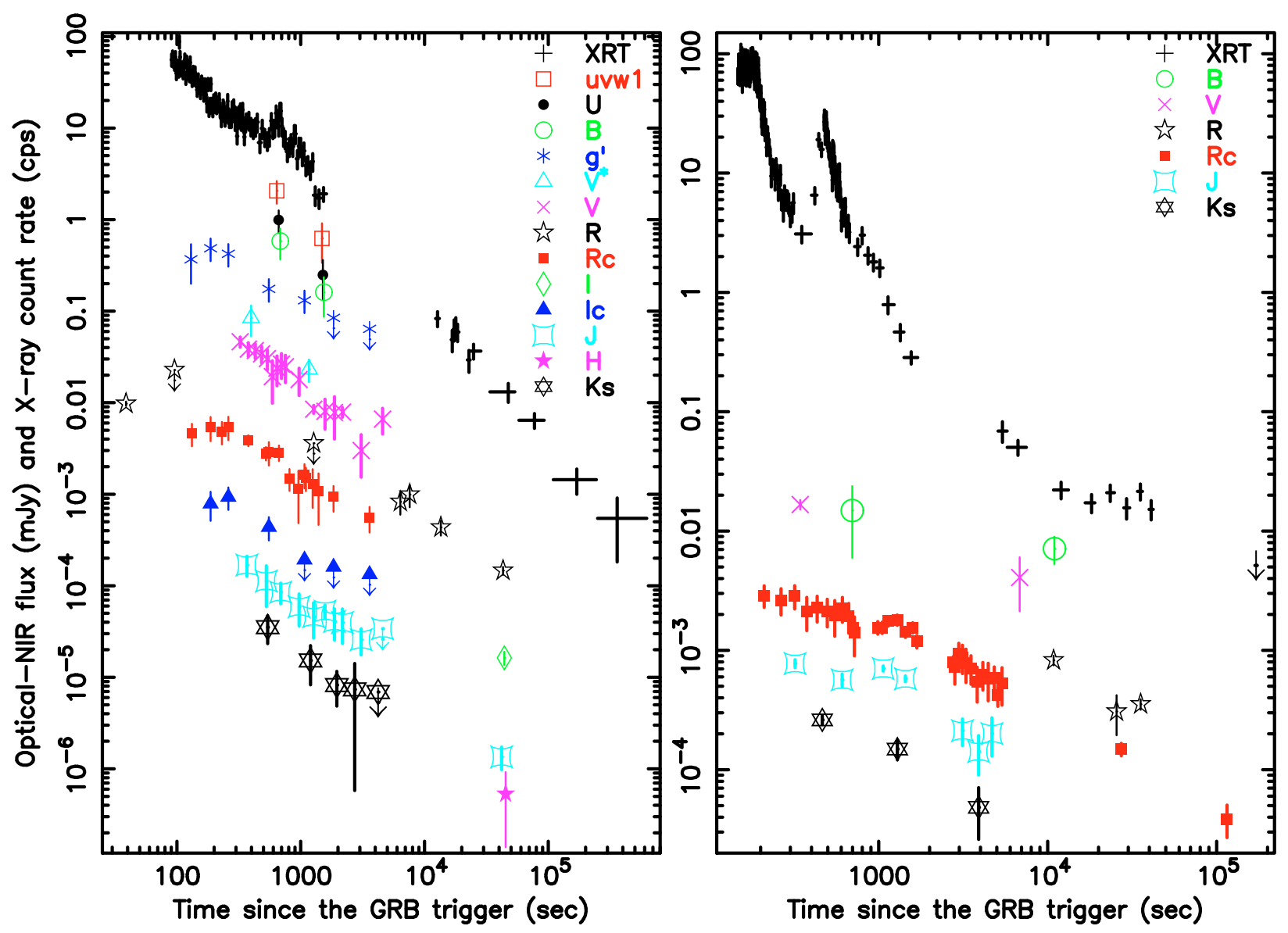

Fig. 1. X-ray and NIR-UV light curves of afterglows of GRB 071112C (left) and GRB 080506 (right). The flux density of the NIR-UV afterglows are shifted by 2 in XRT; $10^{0.5}$ in uvw1- (Oates \& Stratta 2007); $10^{0.0}$ in $U$ - (Oates \& Stratta 2007); $10^{-0.5}$ in $B$ - (Oates \& Stratta 2007); 10 $0^{-1.0}$ in $g^{\prime}$ - (Ishimura et al. 2007; Yoshida et al. 2007); $10^{-1.5}$ in $V^{*}$ (UVOT)- (Oates \& Stratta 2007); $10^{-2.0}$ in $V-; 10^{-2.5}$ in $R$ - (Klotz et al. 2007; Burenin et al. 2007; Chen et al. 2007a; Greco et al. 2007; Minezaki et al. 2007); $10^{-3.0}$ in $R_{\mathrm{C}^{-}}$(Dintinjana et al. 2007; Ishimura et al. 2007; Yoshida et al. 2007); $10^{-3.5}$ in $I$ - (Minezaki et al. 2007); $10^{-4.0}$ in $I_{\mathrm{C}^{-}}$(Ishimura et al. 2007; Yoshida et al. 2007); 10-4.8 in $J$ - (Minezaki et al. 2007); 10 $0^{-5.0}$ in $H$ - (Minezaki et al. 2007); and $10^{-5.5}$ in $K_{\mathrm{s}}$-band (Minezaki et al. 2007) in the left panel. In the right panel, they are shifted by $10^{-0.5}$ in $B$ - (Oates \& Baumgartner 2008); $10^{-1.0}$ in $V$ - (Oates \& Baumgartner 2008); $10^{-1.5}$ in $R$ - (Kocka et al. 2008; de Postigo Ugarte et al. 2008; Sahu et al. 2008; Maeno et al. 2008); $10^{-2.0}$ in $R_{\mathrm{C}^{-}}$(Kann et al. $\left.2008 \mathrm{~b}, \mathrm{a}\right) ; 10^{-2.8}$ in $J-; 10^{-3.1}$ in $K_{\mathrm{s}}$-band.

\section{Results}

\subsection{Optical and X-ray light curves}

In Fig. 1, we show the X-ray and NIR-UV light curves of afterglows of GRB $071112 \mathrm{C}$ (left) and GRB 080506 (right). The X-ray observations by XRT are indicated by the crosses. The other symbols indicate the UV, optical, and NIR observations as described in the figure. In addition to our observations by the Kanata telescope, this figure includes observations reported in GCN Circular: Yuan et al. (2007); Uemura et al. (2007a); Klotz et al. (2007); Burenin et al. (2007); Chen et al. (2007a); Nugent \& Bloom (2007); Dintinjana et al. (2007); Oates \& Stratta (2007); Chen et al. (2007b); Updike et al. (2007); Ishimura et al. (2007); Greco et al. (2007); Sposetti (2007); Yoshida et al. (2007); Uemura et al. (2007b); van der Horst \& Wijers (2007); Chandra \& Frail (2007); Minezaki et al. (2007); Huang et al. (2008) for GRB 071112C and Baumgartner et al. (2008); Kawabata et al. (2008); Osborne et al. (2008); Kann et al. (2008b); Kocka et al. (2008); de Postigo Ugarte et al. (2008); McLean et al. (2008); Oates \& Baumgartner (2008); Sbarufatti et al. (2008); Kann et al. (2008a); Chandra \& Frail (2008); Sahu et al. (2008); Maeno et al. (2008) for GRB 080506. According to Jakobsson et al. (2007a), the host galaxy of GRB 071112C is detected at $R_{\mathrm{c}}=24.5 \pm 0.5$. The host galaxy is so faint that its contribution to the afterglow is negligible in our analysis. The host galaxy of GRB 080506 was not detected.

The X-ray light curve of GRB 071112C can be described with a broken power-law form; $f \propto t^{\alpha_{1}}\left(t \leq t_{\text {break }}\right)$ and, $f \propto$ $t^{\alpha_{2}}\left(t>t_{\text {break }}\right)$ with a weak X-ray flare between $T+450 \mathrm{~s}$ and $T+1270 \mathrm{~s}$. Excluding the X-ray flare phase, we calculated the decay indexes ( $\alpha_{1}$ and $\alpha_{2}$ ), and show them in Table 2 . The break time $\left(t_{\text {break }}\right)$ was estimated to be $7.6 \pm 0.7 \mathrm{ks}$. Table 2 also includes the decay indexes during the rising and decay phases of the X-ray flare which we calculated assuming a single powerlaw form. The optical light curves show a brightening trend before $T+365 \mathrm{~s}$. After the maximum, the flux decayed as a single power-law without a break as observed in the X-ray afterglow. We confirmed that the decay indexes are same in all NIR to UV bands within errors. The decay index was, hence, calculated with all NIR-UV observations, and shown in Table 3.

The X-ray light curve of GRB 080506 (the right panel of Fig. 1) is more complex than that of GRB 071112C. Before $T+200 \mathrm{~s}$, the X-ray flux remained at a high level, which may be related to the prompt emission (McLean et al. 2008; Sbarufatti et al. 2008). A steep decay was observed from $T+200 \mathrm{~s}$ to $T+350 \mathrm{~s}$. Between $T+350 \mathrm{~s}$ and $T+672 \mathrm{~s}$, it showed an X-ray 
Table 2. Decay and spectral index of the X-ray afterglow of GRB 071112C.

\begin{tabular}{cccc}
\hline \hline Region & $T(\mathrm{~s})$ & $\alpha_{\mathrm{X}}^{\dagger}\left(\chi^{2} /\right.$ d.o.f. $)$ & $\beta_{\mathrm{X}}^{\ddagger}$ \\
\hline Normal Decay & $100<T<450,1270<T<1630$ & $-1.27 \pm 0.05(104.8 / 96)$ & $-0.83 \pm 0.04$ \\
Flare Rise & $450<T<650$ & $2.51 \pm 0.77(7.3 / 6)$ & $-0.34 \pm 0.15$ \\
Flare Decay & $650<T<1270$ & $-2.05 \pm 0.26(16.5 / 16)$ & $-0.62 \pm 0.06$ \\
Post-break & $12542<T<147425$ & $-1.43 \pm 0.04(104.8 / 96)$ & $-0.82_{-0.16}^{+0.08}$ \\
\hline
\end{tabular}

Notes. The uncertainties show the $90 \%$ confidence levels of the parameters. ${ }^{(\dagger)}$ X-ray decay index. ${ }^{(\ddagger)}$ X-ray spectral index $\left(\chi^{2} /\right.$ d.o.f. $\left.=587.7 / 298\right)$.

Table 3. Decay indexes of the optical light curves.

\begin{tabular}{llll}
\hline \hline GRB & $T(\mathrm{~s})$ & $\alpha_{\mathrm{O}}^{\dagger}$ & $\chi^{2} /$ d.o.f. \\
\hline GRB 071112C & $250<T<10000$ & $-0.95( \pm 0.02)$ & $31 / 44$ \\
GRB 080506 & $200<T<1300$ & $-0.19( \pm 0.06)$ & $59 / 76$ \\
& $1300<T<2 \times 10^{5}$ & $-0.83( \pm 0.03)$ & \\
\hline
\end{tabular}

Notes. The uncertainties show the $90 \%$ confidence levels of the parameters. ${ }^{(\dagger)}$ Optical decay index.

flare centered at $T+485 \pm 2 \mathrm{~s}$. The X-ray afterglow finally entered a normal decay phase after the X-ray flare. The optical and NIR light curves of GRB 080506 can be described with a broken power-law model. The power-law decay index changes after a break time at $T+1.3 \mathrm{ks}$. The calculated decay indexes are summarized in Tables 3 and 4.

We succeeded in obtaining dense NIR, optical, and UV light curves around X-ray flares both in GRB 071112C and GRB 080506. At the X-ray flare maxima, the X-ray flux increased by a factor of 3.5 and 15 in GRB 071112C and GRB 080506, respectively. On the other hand, the NIR-UV light curves exhibit no significant variations associated with the X-ray flares in both GRBs. The variation amplitudes are $<7 \%$ in $V$ for GRB $071112 \mathrm{C}$, and $<14 \%$ in $R_{\mathrm{C}}$ for GRB 080506.

\subsection{XRT spectral analysis}

In this subsection, we report the result of our analysis on X-ray spectra obtained with XRT, in particular, about the temporal variation of the X-ray spectral index $\left(\beta_{\mathrm{X}}\right)$. Based on the light curve analysis shown in the last section, we defined 4 and 6 phases for GRB 071112C and GRB 080506, as described in Tables 2 and 4, respectively. The following analysis was performed for averaged $\mathrm{X}$-ray spectra of each phase. The average spectra of each phase were fitted with an absorbed power-law model with two absorption components. The first component is the absorption in our galaxy. According to Dickey \& Lockman (1990), the galactic hydrogen column density is $N_{\mathrm{H}}^{\mathrm{gal}}=7.4 \times 10^{20}$ and $1.66 \times 10^{21} \mathrm{~cm}^{2}$ for the direction of GRB 071112C and GRB 080506, respectively. We used those $N_{\mathrm{H}}^{\mathrm{gal}}$ to estimate the galactic absorptions for the GRBs. The second component is the absorption in the host galaxy. The column density, $N_{\mathrm{H}}^{\text {ext }}$, was a free parameter in our analysis.

$N_{\mathrm{H}}^{\text {ext }}$ can be estimated from the absorption model, which depends on the redshift. The redshift correction of the observed energy band is, hence, essential to correct the absorption. We performed the redshift correction to the energy band for GRB 071112C using a reported redshift of $z=0.82$ (Jakobsson et al. 2007b; Cucchiara et al. 2007). About GRB 080506, the redshift correction was not performed since the redshift is not determined. We note that $\beta_{\mathrm{X}}$ is independent of the redshift correction, while $N_{\mathrm{H}}^{\text {ext }}$ depends on it. Using the absorbed power-law model, we confirmed that the column density $N_{\mathrm{H}}^{\text {ext }}$ was constant in all phases for each GRB, while $\beta_{\mathrm{X}}$ changed. We obtained the temporal variation of $\beta_{\mathrm{X}}$ in $0.3-8.0 \mathrm{keV}$ band by simultaneously fitting the spectra of all the phases by letting the $N_{\mathrm{H}}^{\text {ext }}$ to be common among phases and $\beta_{\mathrm{X}}$ to be free for each phase. The obtained $\beta_{\mathrm{X}}$ are summarized in Tables 2 and 4, where the $\chi^{2} /$ d.o.f. of the fitting is $31 / 44$ and 59/76 for GRB 071112 C and GRB 080506, respectively. As shown in the table, the X-ray spectra in the rising phase of the X-ray flares became harder than those in the normal decay phases both in GRB $071112 \mathrm{C}$ and GRB 080506. The column density $N_{\mathrm{H}}^{\text {ext }}$ of GRB $071112 \mathrm{C}$ was estimated to be $7.9 \pm 3.9 \times 10^{20} \mathrm{~cm}^{-2}$.

\section{3. $\alpha-\beta$ relation in the $X$-ray afterglows}

In the standard external shock model, the temporal decay index, $\alpha$, is related to the spectral slope, $\beta$ (e.g. Zhang et al. 2006a). For example, $\alpha$ and $\beta$ in the $\mathrm{X}$-ray regime have a relation of $\alpha_{\mathrm{X}}=1.5 \beta_{\mathrm{X}}$ in the case that the $\mathrm{X}$-ray band is between the synchrotron cooling frequency $v_{\mathrm{c}}$ and the typical frequency $v_{\mathrm{m}}$ under a homogeneous circum-burst medium. The $\alpha$ and $\beta$ during the normal decay phase of GRB $071112 \mathrm{C}$ satisfies this relation. The electron energy distribution index, $p$, was estimated to be $p=2.7$, inferred from $p=1-\frac{4}{3} \alpha_{\mathrm{X}}$ and/or $p=1-2 \beta_{\mathrm{X}}$ (Meszaros \& Rees 1997; Sari et al. 1998). This is similar to a typical $p$ in previously observed afterglows (Liang et al. 2007). On the other hand, the $\alpha_{\mathrm{X}}$ and $\beta_{\mathrm{X}}$ in GRB 080506 do not follow the $\alpha-\beta$ relation.

\subsection{Spectral energy distribution of GRB $071112 C$}

Figure 2 shows NIR-X-ray SEDs of GRB 071112C. The figure contains SEDs at 6 epochs, that is; (a) the rising phase of the optical afterglow $(T+100-T+365 \mathrm{~s})$; (b) the normal decay phase $(T+36-T+450 \mathrm{~s})$; (c) the rising phase of the X-ray flare $(T+450-T+650 \mathrm{~s})$; (d) the decay phase of the X-ray flare $(T+650-T+1270 \mathrm{~s})$; (e) the normal decay phase $(T+1270-T+1630 \mathrm{~s})$; and (f) the post-break phase $(T+12542-T+147425 \mathrm{~s})$. The crosses show the X-ray spectrum. The solid lines indicate the $90 \%$ confidence region of unabsorbed power-law model of the X-ray spectra. The open circles show the observed NIR-UV fluxes. In all phases except for the rising phase of X-ray flare, the NIR-UV fluxes are much lower than those expected from the extrapolated power-law components of the X-ray spectra. Thus, the NIR-UV fluxes are definitely reduced by the interstellar extinction.

We estimated the extinction in the $V$-band, $A_{V}$, from $N_{\mathrm{H}}$ obtained from the X-ray spectral analysis. The ratio, $N_{\mathrm{H}} / A_{V}$, has been reported in our and nearby galaxies, that is, $N_{\mathrm{H}} / A_{V}=$ $1.6 \times 10^{21} \mathrm{~cm}^{-2}$ for Milky Way (MW), $7.6 \times 10^{21} \mathrm{~cm}^{-2}$ for 
Table 4. Decay and spectral index of the X-ray afterglow of GRB 080506.

\begin{tabular}{cccc}
\hline \hline Region & $T(\mathrm{~s})$ & $\alpha_{\mathrm{X}}^{\dagger}\left(\chi^{2} /\right.$ d.o.f. $)$ & $\beta_{\mathrm{X}}^{\ddagger}$ \\
\hline Prompt Emission & $145-200$ & prompt like & $-0.38 \pm 0.03$ \\
Steep Decay & $200-350$ & $-4.8 \pm 0.2(43.3 / 30)$ & $-1.05 \pm 0.06$ \\
Flare Rise & $350-474$ & $10.14 \pm 0.72(23.8 / 6)$ & $-0.80 \pm 0.18$ \\
Flare Peak & $474-503$ & - & $-1.02 \pm 0.13$ \\
Flare Decay & $503-672$ & $-6.41 \pm 0.24(50.9 / 47)$ & $-1.49 \pm 0.10$ \\
Normal Decay & $10900-70300$ & $-0.81 \pm 0.05(55.7 / 19)$ & $-1.26_{-0.16}^{+0.17}$ \\
\hline
\end{tabular}

Notes. The uncertainties show the $90 \%$ confidence levels of the parameters. ${ }^{(\dagger)}$ X-ray decay index. ${ }^{(\ddagger)}$ X-ray spectral index $\left(\chi^{2} /\right.$ d.o.f. $\left.=587.7 / 298\right)$.

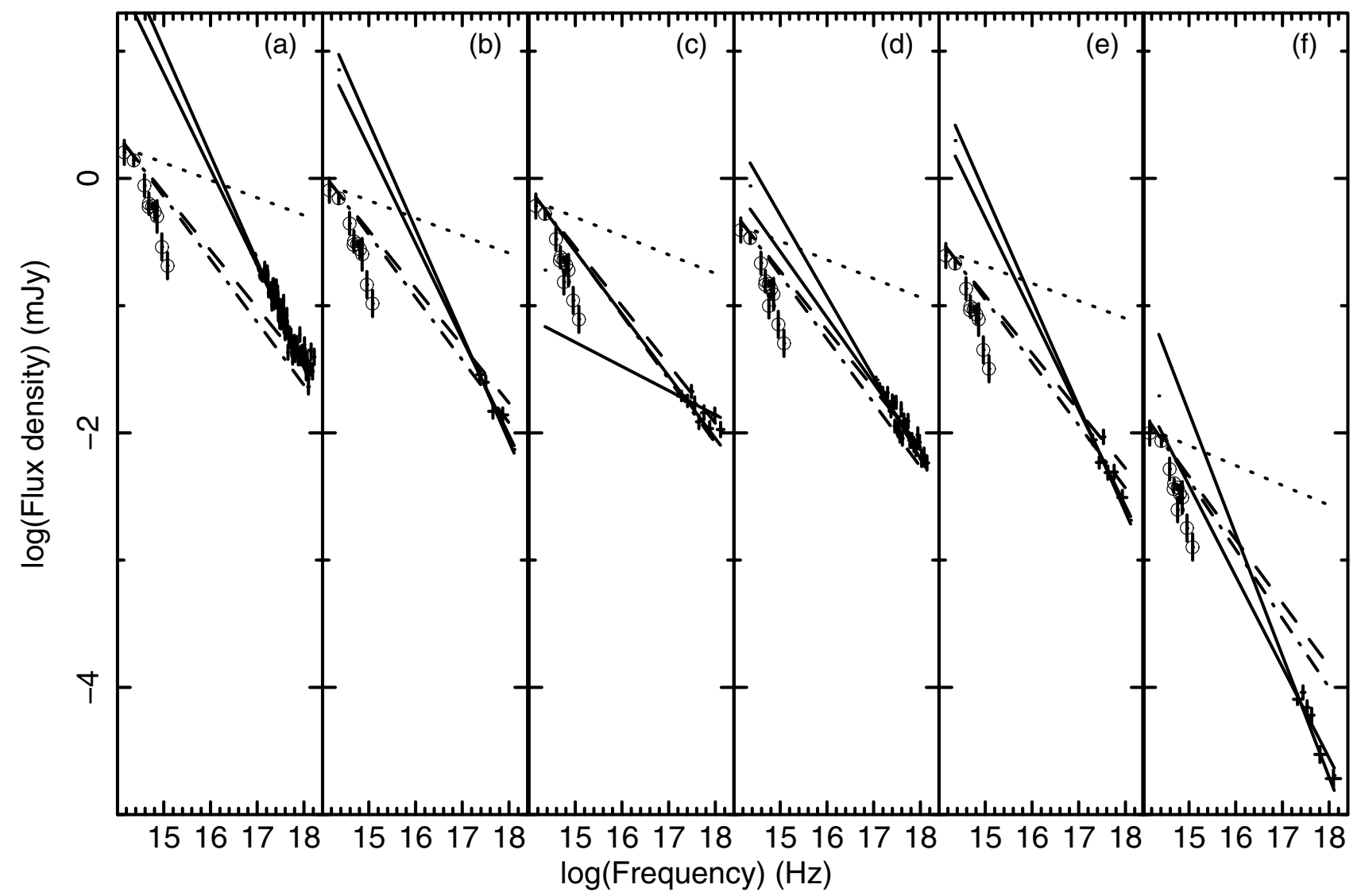

Fig. 2. SEDs of the NIR-X-ray regime of GRB 071112C. The 6 panels show the SED on a) $T+100-T+365 \mathrm{~s}$; b) $T+365-T+450 \mathrm{~s}$; c) $T+450-T+650 \mathrm{~s}$; d) $T+650-T+1270 \mathrm{~s}$; e) $T+1270-T+1630 \mathrm{~s}$; and f) $T+12542-T+147425 \mathrm{~s}$. The crosses show the X-ray spectra. The solid lines in the figure indicate the $90 \%$ fitting error region unabsorbed power-law model of the X-ray spectra. The open circles show observed infrared-UV SED. The doted, dashed, and dash-doted lines indicate the best-fitted power-law models of the NIR-UV SEDs calculated with the extinction model in the MW, LMC, and SMC, respectively.

the Large Magellanic Cloud (LMC), and $1.5 \times 10^{22} \mathrm{~cm}^{-2}$ for the Small Magellanic Cloud (SMC) (Pei 1992; Weingartner \& Draine 2000). Using those 3 models, we corrected the extinction in the $V$-band. The conversion from $A_{V}$ to $A_{\lambda}$ of the other bands was performed based on Cardelli et al. (1989). In Fig. 2, the dotted, dashed, and dash-dotted lines indicate the best-fitted powerlaw models for the corrected NIR-UV SEDs with the MW, LMC, and SMC models, respectively. In the case that the NIR-UV extinction was corrected with the LMC or SMC model, the X-ray flux has an excess over the SED extrapolated from the NIR-UV regime in panel (a) of Fig. 2. Such an X-ray excess is inconsistent with the standard external shock model. In the case that the extinction model is the MW model, the SED should have a break between the optical and UV band in panel (f), while such a break is not clearly seen in the observed NIR-UV SED. However, the break could be in UV-X-ray SED, when considering the error of $\mathrm{X}$-ray spectral index.

In the framework of the GRB external shock model, NIR$\mathrm{X}$-ray SEDs can be described with a single power-law model if the cooling frequency, $v_{\mathrm{c}}$, is below the NIR band. Alternatively, SEDs have a break in the case that $v_{\mathrm{c}}$ lies between the NIRX-ray bands. As mentioned above and shown in Fig. 2, a single power-law model cannot reproduce the observed SED of the NIR-X-ray bands regardless of the ambiguity of the extinction. Therefore, the standard external shock model can explain the observed SED only when $v_{\mathrm{c}}$ lies between the NIR-X-ray bands. Then, the SED should have a break between the NIR-X-ray bands. This break actually appears in the case that $N_{\mathrm{H}}^{\text {ext }} / A_{V}$ in 


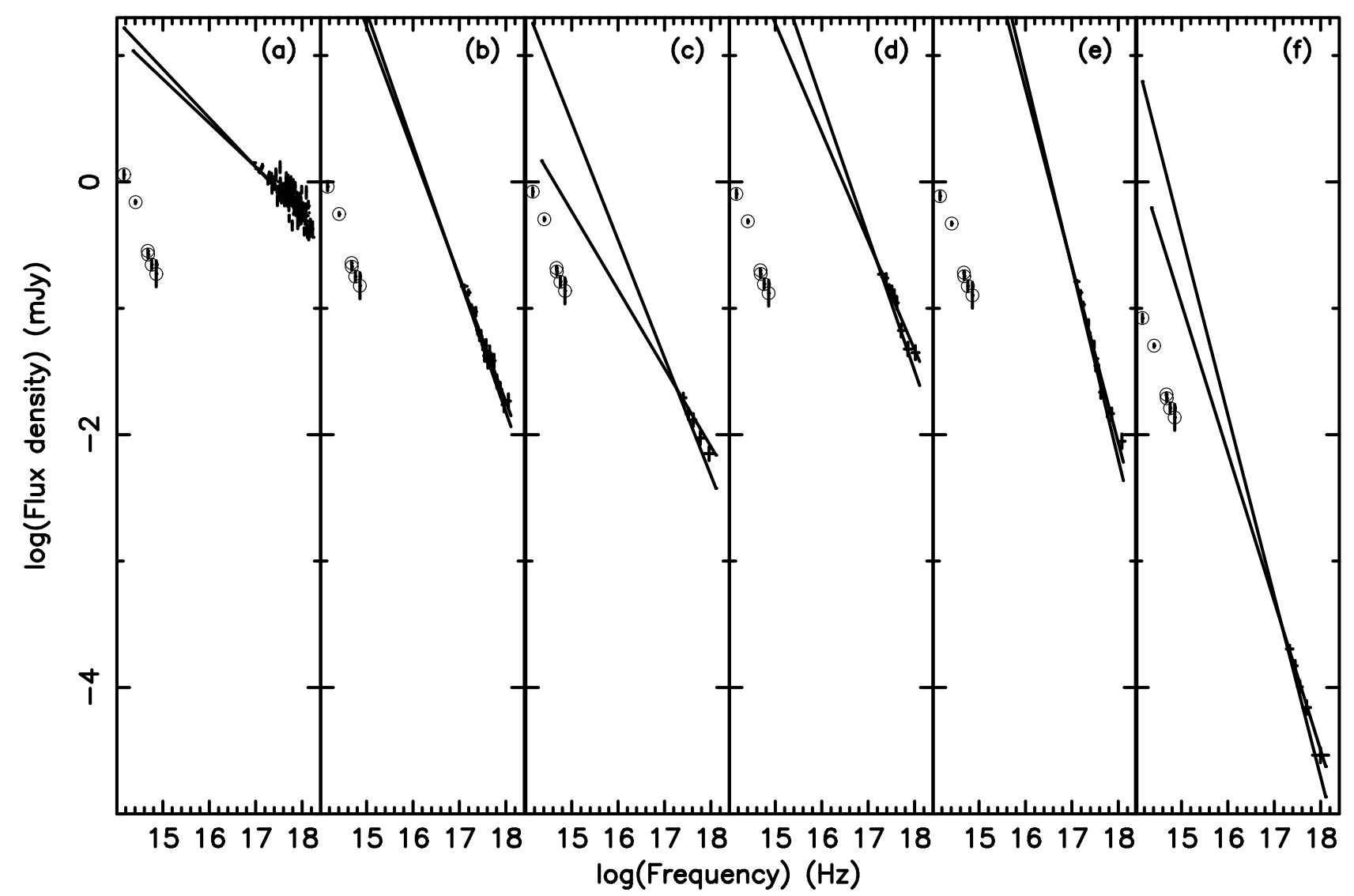

Fig. 3. SEDs of the NIR-X-ray regime of GRB 080506. These epochs correspond to the phases defined in Table 4, that is, a) Prompt Emission on $T+145-T+200 \mathrm{~s}$; b) Steep Decay on $T+200-T+350 \mathrm{~s}$; c) Flare Rise on $T+350-T+474 \mathrm{~s}$; d) Flare Peak on $T+474-T+503 \mathrm{~s}$; e) Flare Decay on $T+503-T+672 \mathrm{~s}$; and f) Normal Decay on $T+10900-T+70300 \mathrm{~s}$. The solid lines show $90 \%$ fitting error region of X-ray spectra that corrected for interstellar extinction. No redshift correction was performed to the energy band.

the host galaxy of GRB $071112 \mathrm{C}$ is between the values of the MW and LMC models.

\subsection{Spectral energy distribution of GRB 080506}

These epochs correspond to the phases defined in Table 4, that is, (a) the prompt emission phase $(T+145-T+200 \mathrm{~s})$, (b) the steep decay phase $(T+200-T+350 \mathrm{~s})$, (c) the flare rise phase $(T+350-T+474 \mathrm{~s})$, (d) the flare peak phase $(T+474-T+503 \mathrm{~s})$, (e) the flare decay phase $(T+503-T+672 \mathrm{~s})$, and (f) the normal decay phase $(T+10900-T+70300 \mathrm{~s})$. As can be seen in the figure, the spectral slope of X-rays clearly changed, while no significant change was detected in the optical-NIR range during the X-ray flare phase from panel (c) to (e).

As well as the case of GRB 071112C, the NIR-UV fluxes are much lower than those expected from the X-ray spectra indicated by the solid line in the figure. Thus, the observed NIR-UV fluxes were definitely reduced by the interstellar extinction in the host galaxy also in the case of GRB 080506. Since the lack of a measured redshift prevents us from determining $N_{\mathrm{H}}$ based on the X-ray spectrum, we cannot apply the same approach for the absorption correction to GRB 080506, as in GRB 071112C.

In the framework of the external shock model, the condition of $\alpha_{\mathrm{opt}}=\alpha_{\mathrm{X}}$ means that $v_{\mathrm{c}}$ is below the optical frequency, and hence, an optical-X-ray SED should be described with a single power-law, namely $\beta_{\text {opt }}=\beta_{\mathrm{X}}$. In the case of GRB 080506, the observed $\alpha_{\text {opt }}$ was in agreement with $\alpha_{\mathrm{X}}$ within errors after $T+1.5 \mathrm{ks}$. Hence, the standard external shock model predicts $\beta_{\mathrm{opt}}=\beta_{\mathrm{X}}$ in GRB 080506 after $T+1.5 \mathrm{ks}$. We calculated $\beta_{\text {opt }}=0.95 \pm 0.05$ shown by dotted line in panel (f) of Fig. 3, using the NIR-UV data after $T+1.5 \mathrm{ks}$ in which the galactic extinction was corrected (Schlegel et al. 1998). This is, however, significantly different from $\beta_{\mathrm{X}}$ in the normal decay phase, as shown in Table 4. This is possibly due to a significant reddening of the optical afterglow in the host galaxy of the GRB.

Then, we can estimate the extinction in the host galaxy in the NIR-UV range, assuming a single power-law SED between the NIR-X-ray range. We defined the extinction, $A_{\lambda}$, as $A_{v}=F_{\lambda}^{0} / F_{\lambda}^{\mathrm{obs}}$, where $F_{\lambda}^{0}$ is the flux extrapolated from the X-ray power-law spectrum, $F_{v}^{0}=F_{\mathrm{X}}\left(v / v_{\mathrm{X}}\right)^{\beta}$ where $F_{\mathrm{X}}$ the observed X-ray band $\left(v_{\mathrm{X}}\right)$ flux. $F_{\lambda}^{\text {obs }}$ is the observed NIR-UV fluxes. Figure 4 shows the obtained extinction curve. The extinction $A_{\lambda}$ was normalized at $A_{V}$. The extinction curves in the MW is also shown for comparison in Figs. 4 (Cardelli et al. 1989). In general, the extinction is larger in the UV region rather than the NIR one. In the case of GRB 080506, however, the extinction is larger in the NIR than in the UV region. This result is problematic for the standard extinction model with dusts (Cardelli et al. 1989). Thus, the extraordinary extinction curve in Fig. 4 indicates $\beta_{\mathrm{opt}} \neq \beta_{\mathrm{X}}$ in the normal decay phase of GRB 080506, while $\alpha_{\text {opt }}=\alpha_{\mathrm{X}}$. In the case that we correct the flux with a general extinction law, the SED definitely has a break between the NIR and X-ray bands since the observed $\beta_{\text {opt }}$ is smaller than $\beta_{\mathrm{X}}$. As well as the $\alpha_{\mathrm{X}}-\beta_{\mathrm{X}}$ relation reported in Sect. 3.3, the condition in GRB 080506 is inconsistent with the standard external shock model. 
T. Uehara et al.: Infrared/optical - X-ray simultaneous observations of X-ray flares in GRB 071112C and GRB 080506

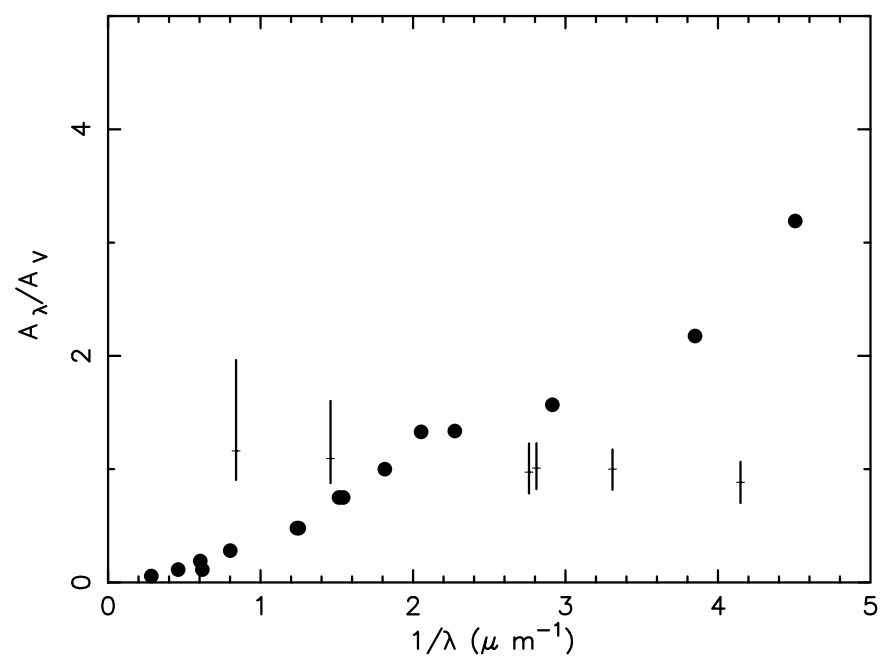

Fig. 4. Extinction curves (normalized at the $V$ band) for the host galaxy of GRB 080506 estimated with a simple power-law SED extrapolated from the X-ray region. The bar and filled circles show the curves for GRB 080506 and MW.

\section{Discussion}

In this section, we discuss whether the X-ray flare originates in the external or internal shocks.

If they arise from external shocks, optical and NIR flares should be contemporaneously detected during the X-ray flares (Fan et al. 2005). In addition, the spectral index in the optical band should also change (e.g. Zhang et al. 2006a). Our observations unambiguously show no optical variation associated with the X-ray flares. The lack of optical variations is consistent with previously reported observations (e.g. Burrows et al. 2005; Krimm et al. 2007; Butler \& Kocevski 2007). The optical spectral index also unchanged during the X-ray flares in both GRB 071112C and 080506. Therefore, the optical behavior during these X-ray flares is inconsistent with the prediction from the standard external shock model. On the other hand, the late internal shock model can explain the X-ray flare even if no contemporaneous flares are observed in the optical and NIR bands (Burrows et al. 2005). In fact, the lack of the NIR-optical variations in both GRB $071112 \mathrm{C}$ and GRB 080506 is consistent with this model.

In the following, we propose an alternative model for the $\mathrm{X}$-ray flare. We consider two components of the external shocks; one originates in the main shell which produces the normal afterglow, and the other originates in a delayed shell which is responsible for the X-ray flare. The observed sharp decay of the $\mathrm{X}$-ray flare can be explained by this scenario because the decay slope of the emission from the delayed shell can be apparently steep in the time frame of the normal afterglow (Zhang et al. 2006b; Yamazaki et al. 2006). The delayed shell could generate a prominent X-ray emission under the condition that it passes a region containing enough interstellar medium. Such a condition is probably achieved, for example, if the opening angle of the delayed jet is larger than that of the main jet, or if the axis of the delayed jet is off to that of the main jet. These conditions were originally proposed to explain "X-ray flashes", which are analogous to GRBs except for their softer emission and less energetics (Yamazaki et al. 2002, 2003, 2004; Lamb et al. 2005; Li 2006). Piro et al. (2005) and Galli \& Piro (2006) have proposed models for the X-ray flare, in which the delayed shell interacts with the reverse shock region of the preceding jet.
In our model, we assume that the temporal evolution of the emission from the delayed shell is the same as that of the main shell, following the standard external shock model (Sari et al. 1998). For the decay phase of the X-ray flare, we assumed that the spectrum of delayed shell overlaps that of the main shell. We denote the maximum and cooling frequencies of the synchrotron emission as $v_{\mathrm{m}}$ and $v_{\mathrm{c}}$ for the main shell, and $v_{\mathrm{m}}^{\prime}$ and $v_{\mathrm{c}}^{\prime}$ for the delayed shell. Then, we investigated several conditions of $v_{\mathrm{m}}^{\prime}$ and $v_{\mathrm{c}}^{\prime}$, which satisfy the observed amplitudes of variations and electron energy distribution index.

We have mentioned in Sects. 2.4 and 2.5 that for both events we analyzed, the SEDs of the normal afterglow component are expected to have a break between the NIR and X-ray bands. This break means that $v_{\mathrm{c}}$ lies in this bands. There is no spectral break in the observed NIR-optical SEDs and X-ray spectra. Therefore, $v_{\mathrm{c}}$ are constrained as $6 \times 10^{14} \mathrm{~Hz}<v_{\mathrm{c}}<2 \times 10^{17} \mathrm{~Hz}$ and $5 \times$ $10^{14} \mathrm{~Hz}<v_{\mathrm{c}}<2 \times 10^{16} \mathrm{~Hz}$ for GRB $071112 \mathrm{C}$ and GRB 080506, respectively. The above frequencies are those in the observer's frame. Because we focus on the ratio of the frequency in the following discussion, the redshift correction to the energy band is not important.

The cooling frequency of the normal afterglow $v_{\mathrm{c}}$, is also constrained using the observed amplitudes of the X-ray flares. In this procedure, we consider ten cases depending on the relationship among $v_{\mathrm{m}}^{\prime}, v_{\mathrm{c}}^{\prime}$, and the observation frequencies of $v_{\mathrm{O}}$ and $v_{\mathrm{X}}$. As listed in Table 5, possible conditions are divided into five cases in the slow cooling regime $\left(v_{\mathrm{m}}^{\prime}<v_{\mathrm{c}}^{\prime}\right)$ and five cases in the fast cooling regime $\left(v_{\mathrm{c}}^{\prime}<v_{\mathrm{m}}^{\prime}\right)$. Since we discuss the SED of the decay phase of the X-ray flare, the conditions are limited to those ten cases; the conditions of $v_{\mathrm{m}}^{\prime}<v_{\mathrm{X}}$ and $v_{\mathrm{c}}^{\prime}<v_{\mathrm{X}}$ are required for the decay phase in the slow and fast cooling regime, respectively. The cases for the slow and fast cooling regimes are indicated by the characters " $S$ " and "F", respectively.

For example, in case S1 in Table 5, the X-ray and optical flux densities of the normal decay component, $F_{\mathrm{n}, \mathrm{X}}$ and $F_{\mathrm{n}, \mathrm{O}}$ are related as;

$F_{\mathrm{n}, \mathrm{X}}=F_{\mathrm{n}, \mathrm{O}}\left(\frac{v_{\mathrm{c}}}{v_{\mathrm{O}}}\right)^{-\frac{p-1}{2}}\left(\frac{v_{\mathrm{X}}}{v_{\mathrm{c}}}\right)^{-\frac{p}{2}}$.

Here we assumed an SED predicted by the standard external shock model (Sari et al. 1998). Similarly, the optical and X-ray flux densities of the X-ray flare component, $F_{\text {flare, }}$ and $F_{\text {flare, }}$ are related as;

$F_{\text {flare }, \mathrm{O}}=F_{\text {flare }, \mathrm{X}}\left(\frac{v_{\mathrm{c}}^{\prime}}{v_{\mathrm{X}}}\right)^{-\frac{p^{\prime}}{2}}\left(\frac{v_{\mathrm{O}}}{v_{\mathrm{c}}^{\prime}}\right)^{-\frac{p^{\prime}-1}{2}}$,

where $p^{\prime}$ is the electron distribution index of the X-ray flare component. We define the ratios of the flare to the normal fluxes in the X-ray and optical bands as

$A=F_{\text {flare }, \mathrm{X}} / F_{\mathrm{n}, \mathrm{X}}$,

$a=F_{\text {flare }, \mathrm{O}} / F_{\mathrm{n}, \mathrm{O}}$.

Using Eqs. (1)-(4), we obtain

$v_{\mathrm{c}}=\left(\frac{a}{A}\right)^{2} 10^{3\left(p-p^{\prime}\right)} v_{\mathrm{c}}^{\prime}$

where we take $v_{\mathrm{X}} / v_{\mathrm{O}} \approx 10^{3}$. In this case $\mathrm{S} 1$, the cooling frequency of the flare component, $v_{\mathrm{c}}^{\prime}$, is assumed to lie between $v_{\mathrm{O}}$ and $v_{\mathrm{X}}$. Given this fact, and substituting observed quantities summarized in Table 6 into Eq. (5), we derive the condition 
Table 5. $v_{\mathrm{c}}$ estimated for possible cases on $v_{\mathrm{m}}^{\prime}$ and $v_{\mathrm{c}}^{\prime}$ in the two-component external shock model.

\begin{tabular}{lllcc}
\hline \hline Case & Condition & Formula & $v_{\mathrm{c}}$ (Hz) \\
& & GRB 071112C & GRB 080506 \\
\hline S1 & $v_{\mathrm{m}}^{\prime}<v_{\mathrm{O}}<v_{\mathrm{c}}^{\prime}<v_{\mathrm{X}}$ & $\left(\frac{a}{A}\right)^{2} 10^{3\left(p-p^{\prime}\right)} v_{\mathrm{c}}^{\prime}$ & $<1 \times 10^{14}$ & $<1 \times 10^{10}$ \\
S2 & $v_{\mathrm{m}}^{\prime}<v_{\mathrm{O}}<v_{\mathrm{X}}<v_{\mathrm{c}}^{\prime}$ & $\left(\frac{a}{A}\right)^{2} 10^{3\left(p-p^{\prime}\right)+18}$ & $<6 \times 10^{14} \sqrt{ }$ & $<5 \times 10^{11}$ \\
S3 & $v_{\mathrm{O}}<v_{\mathrm{m}}^{\prime}<v_{\mathrm{c}}^{\prime}<v_{\mathrm{X}}$ & $\left(\frac{a}{A}\right)^{2} 10^{3 p-18 p^{\prime}+5} v_{\mathrm{m}}^{\prime p^{\prime}-1} v_{\mathrm{c}}^{\prime 5 / 3}$ & see Fig. $5 \sqrt{ }$ & see Fig. 5 \\
S4 & $v_{\mathrm{O}}<v_{\mathrm{m}}^{\prime}<v_{\mathrm{X}}<v_{\mathrm{c}}^{\prime}$ & $\left(\frac{a}{A}\right)^{2} 10^{3 p-18 p^{\prime}+23} v_{\mathrm{c}}^{\prime}$ & $<3 \times 10^{17} \sqrt{ }$ & $<3 \times 10^{15} \sqrt{ }$ \\
S5 & $v_{\mathrm{m}}^{\prime}<v_{\mathrm{c}}^{\prime}<v_{\mathrm{O}}<v_{\mathrm{X}}$ & $\left(\frac{a}{A}\right)^{2} 10^{33\left(p-p^{\prime}\right)+15}$ & $<3 \times 10^{-8}$ & $<2$ \\
F1 & $v_{\mathrm{c}}^{\prime}<v_{\mathrm{O}}<v_{\mathrm{m}}^{\prime}<v_{\mathrm{X}}$ & $\left(\frac{a}{A}\right)^{2} 10^{3 p-9 p^{\prime}+30} v_{\mathrm{c}}^{\prime\left(p^{\prime}-1\right)}$ & $<1 \times 10^{22} \sqrt{ }$ & $<5 \times 10^{36} \sqrt{ }$ \\
F2 & $v_{\mathrm{c}}^{\prime}<v_{\mathrm{O}}<v_{\mathrm{X}}<v_{\mathrm{m}}^{\prime}$ & $\left(\frac{a}{A}\right)^{2} 10^{3 p+12}$ & $<2 \times 10^{13}$ & $<1 \times 10^{15} \sqrt{ }$ \\
F3 & $v_{\mathrm{O}}<v_{\mathrm{c}}^{\prime}<v_{\mathrm{m}}^{\prime}<v_{\mathrm{X}}$ & $\left(\frac{a}{A}\right)^{2} 10^{3 p-18 p^{\prime}+5} v_{\mathrm{m}}^{p^{\prime}-1 / 3} v_{\mathrm{c}}^{\prime}$ & see Fig. $5 \sqrt{ }$ & see Fig. 5 \\
F4 & $v_{\mathrm{O}}<v_{\mathrm{c}}^{\prime}<v_{\mathrm{X}}<v_{\mathrm{m}}^{\prime}$ & $\left(\frac{a}{A}\right)^{2} 10^{3 p-18 p^{\prime}+5} v_{\mathrm{c}}^{\prime p^{\prime}+2 / 3}$ & $<5 \times 10^{16} \sqrt{ }$ & $<6 \times 10^{13}$ \\
F5 & $v_{\mathrm{c}}^{\prime}<v_{\mathrm{m}}^{\prime}<v_{\mathrm{O}}<v_{\mathrm{X}}$ & $\left(\frac{a}{A}\right)^{2} 10^{33\left(p-p^{\prime}\right)+15} v_{\mathrm{m}}^{\prime}$ & $<3 \times 10^{-8}$ & $<4 \times 10^{13}$ \\
\hline
\end{tabular}

Notes. The checks $(\sqrt{ })$ show the cases that the present model can reproduce the observed SED variations.
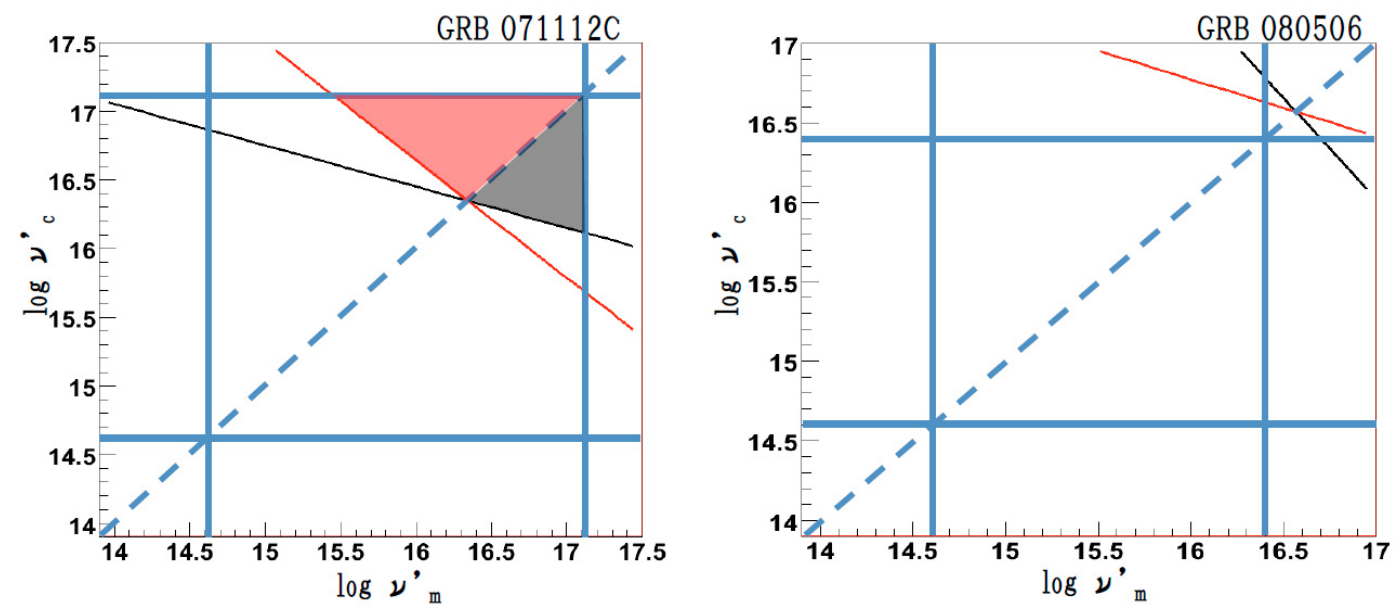

Fig. 5. Allowed region of $v_{c}^{\prime}$ and $v_{m}^{\prime}$. The red and gray shaded regions show the areas in case F3 and S3, respectively. The blue lines show the assumed condition for $v_{\mathrm{c}}^{\prime}$ and $v_{\mathrm{m}}^{\prime}$. These frequencies are $6 \times 10^{14} \mathrm{~Hz}-2 \times 10^{17} \mathrm{~Hz}$ and $5 \times 10^{14} \mathrm{~Hz}-2 \times 10^{16} \mathrm{~Hz}$ for GRB $071112 \mathrm{C}$ and GRB 080506 , respectively. Left and right panels show the cases of GRB 071112C and GRB 080506, respectively.

for the cooling frequency of the normal afterglow component as $v_{\mathrm{c}}<1 \times 10^{14}$ and $<4 \times 10^{10} \mathrm{~Hz}$ for GRB $071112 \mathrm{C}$ and GRB 080506, respectively. Those $v_{\mathrm{c}}$ was calculated taking into account the uncertainties of $p$ and $p$ ' in order to obtain firm estimates. Both for GRB 071112C and 080506, two independent conditions give no allowed regions for $v_{\mathrm{c}}$, so that case $\mathrm{S} 1$ fails to explain the observed results.

Similar to case $\mathrm{S} 1, v_{\mathrm{c}}$ in the other nine cases are also evaluated. Table 5 shows the results. Upper limits of $v_{\mathrm{c}}$ are given in the table if it is independent of $v_{\mathrm{c}}^{\prime}$ and $v_{\mathrm{m}}^{\prime}$ or if it is a function of either of them (cases S1, S2, S4, F1, F2, and F4). If $v_{\mathrm{c}}$ depends on both $v_{\mathrm{c}}^{\prime}$ and $v_{\mathrm{m}}^{\prime}$ (cases S3 and F3), then in the $v_{\mathrm{c}}^{\prime}-v_{\mathrm{m}}^{\prime}$ plane we search for allowed regions to satisfy the conditions of $6 \times 10^{14} \mathrm{~Hz}<v_{\mathrm{c}}<2 \times 10^{17} \mathrm{~Hz}$ and $5 \times 10^{14} \mathrm{~Hz}<v_{\mathrm{c}}<2 \times 10^{16} \mathrm{~Hz}$ for GRB 071112C and GRB 080506, respectively. Figure 5 shows the results. The left and right panels of Fig. 5 are for GRB 071112C and GRB 080506, respectively. The blue solid and dotted lines indicate the assumed conditions for $v_{\mathrm{c}}^{\prime}$ and $v_{\mathrm{m}}^{\prime}$. The red and gray shaded regions indicate the allowed region for $v_{\mathrm{c}}$ in cases F3 and S3, respectively. Thus, both cases F3 and $\mathrm{S} 3$ can explain the observation with the two-component external
Table 6. Observational parameters used in the two-component external shock model.

\begin{tabular}{lll}
\hline \hline results & GRB 071112C & GRB 080506 \\
\hline$A$ & 3.5 & 15 \\
$a$ & $<0.07$ & $<0.14$ \\
$p$ & $1.66( \pm 0.10)$ & $2.70( \pm 0.34)$ \\
$p^{\prime}$ & $1.24( \pm 0.26)$ & $2.92( \pm 0.20)$ \\
\hline
\end{tabular}

shock model for only GRB 071112C. On the other hand, there is no allowed region in the case of GRB 080506, as can be seen in the right panel of Fig. 5. The "check" symbols are given in Table 5 in the case that there are allowed values of parameters that can reproduce the observed SED variations. For both GRBs, there are several cases in which the observations can be explained by our model.

Note that all parameters needed for the above discussion are the variation amplitudes during the X-ray flares in the optical and $\mathrm{X}$-ray ranges, $a$ and $A$, and electron energy distribution index, $p$ and $p^{\prime}$. Thus, the discussion was independent of the uncertainty in the correction of the dust extinction in the GRB host galaxies. 


\section{Conclusion}

We have observed GRB $071112 \mathrm{C}$ and GRB 080506 before the start of X-ray flares. In conjunction with published X-ray and optical data, we analyzed densely sampled light curves of the early afterglows and SEDs of the NIR-X-ray range. We found that the SEDs in the normal decay phase had a break between the UV and soft X-ray regions. No significant variation in the optical-NIR range was detected contemporaneous to the X-ray flares. The lack of the optical-NIR variation suggests that the late internal shock is a reasonable origin for the X-ray flare. In addition, we found that two-component external shock model can also explain the observed variations of SEDs during the X-ray flares.

Acknowledgements. The authors thank the referee for careful reading and many useful comments. The authors also thank the Swift team for development of hardware/software and operation. SN is supported by Research Fellowships of the Japan Society for the Promotion of Science for Young Scientists.

\section{References}

Baumgartner, W. H., Burrows, D. N., Chester, M. M., et al. 2008, GRB Coordinates Network, 7685, 1

Burenin, R., Khamitov, I., Pavlinsky, M., et al. 2007, GRB Coordinates Network, 7066, 1

Burrows, D. N., Romano, P., Falcone, A., et al. 2005, Science, 309, 1833

Butler, N. R., \& Kocevski, D. 2007, ApJ, 663, 407

Cardelli, J. A., Clayton, G. C., \& Mathis, J. S. 1989, ApJ, 345, 245

Chandra, P., \& Frail, D. A. 2007, GRB Coordinates Network, 7096, 1

Chandra, P., \& Frail, D. A. 2008, GRB Coordinates Network, 7698, 1

Chen, S. L., Li, A., \& Wei, D. M. 2006, ApJ, 647, L13

Chen, I. C., Huang, K. Y., \& Urata, Y. 2007a, GRB Coordinates Network, 7067, 1

Chen, I. C., Huang, K. Y., \& Urata, Y. 2007b, GRB Coordinates Network, 7083,

Chincarini, G., Moretti, A., Romano, P., et al. 2007, ApJ, 671, 1903

Chincarini, G., Mao, J., Margutti, R., et al. 2010, MNRAS, submitted [arXiv: 1004.0901$]$

Costa, E., Frontera, F., Heise, J., et al. 1997, Nature, 387, 783

Cucchiara, A., Fox, D. B., Berger, E., \& Perri, M. 2007, GRB Coordinates Network, 7086, 1

de Postigo Ugarte, A., Thoene, C. C., Fynbo, J. P. U., Leitet, E., \& Trinchant, L. M. 2008, GRB Coordinates Network, 7690, 1

Della Valle, M., Malesani, D., Benetti, S., et al. 2003, A\&A, 406, L33

Dickey, J. M., \& Lockman, F. J. 1990, ARA\&A, 28, 215

Dintinjana, B., Maticic, S., Mikuz, H., \& Skvarc, J. 2007, GRB Coordinates Network, 7078, 1

Falcone, A. D., Morris, D., Racusin, J., et al. 2007, ApJ, 671, 1921

Fan, Y. Z., Zhang, B., \& Wei, D. M. 2005, ApJ, 628, L25

Frail, D. A., Kulkarni, S. R., Nicastro, L., Feroci, M., \& Taylor, G. B. 1997, Nature, 389, 261

Galli, A., \& Piro, L. 2006, A\&A, 455, 413

Greco, G., Terra, F., Bartolini, C., et al. 2007, GRB Coordinates Network, 7089,

Hjorth, J., Sollerman, J., Møller, P., et al. 2003, Nature, 423, 847

Huang, K. Y., Schwamb, M. E., Wang, J. H., Wen, C. Y., \& Survey, T. A. O. 2008, GRB Coordinates Network, 7334, 1

Ioka, K., Kobayashi, S., \& Zhang, B. 2005, ApJ, 631, 429

Ishimura, T., Shimokawabe, T., Mori, Y., et al. 2007, GRB Coordinates Network, 7087, 1

Jakobsson, P., Fynbo, J. P. U., Malesani, D., et al. 2007a, GRB Coordinates Network, 7088, 1

Jakobsson, P., Fynbo, J. P. U., Vreeswijk, P. M., Malesani, D., \& Sollerman, J. 2007b, GRB Coordinates Network, 7076, 1

Kann, D. A., Klose, S., \& Zeh, A. 2006, ApJ, 641, 993

Kann, D. A., Hoegner, C., \& Ertel, S. 2008a, GRB Coordinates Network, 7696,
Kann, D. A., Hoegner, C., \& Ertel, S. 2008b, GRB Coordinates Network, 7688, 1

Kawabata, K. S., Sasada, M., Uemura, M., et al. 2008, GRB Coordinates Network, 7686, 1

Klotz, A., Boer, M., \& Atteia, J. L. 2007, GRB Coordinates Network, 7065, 1

Kocka, M., Nekola, M., Strobl, J., et al. 2008, GRB Coordinates Network, 7689,

Krimm, H. A., Granot, J., Marshall, F. E., et al. 2007, ApJ, 665, 554

Lamb, D. Q., Donaghy, T. Q., \& Graziani, C. 2005, ApJ, 620, 355

Li, L.-X. 2006, MNRAS, 372, 1357

Liang, E.-W., Zhang, B.-B., \& Zhang, B. 2007, ApJ, 670, 565

Maeno, S., Naito, H., Narusawa, S., \& Iizuka, R. 2008, GRB Coordinates Network, 7709, 1

Malesani, D., Tagliaferri, G., Chincarini, G., et al. 2004, ApJ, 609, L5

McLean, K., Barthelmy, S. D., Baumgartner, W., et al. 2008, GRB Coordinates Network, 7692, 1

Meszaros, P., \& Rees, M. J. 1997, ApJ, 482, L29

Minezaki, T., Price, P. A., Yoshii, Y., \& Cowie, L. L. 2007, GRB Coordinates Network, 7135, 1

Nugent, P., \& Bloom, J. S. 2007, GRB Coordinates Network, 7069,

Oates, S. R., \& Baumgartner, W. 2008, GRB Coordinates Network, 7693, 1

Oates, S. R., \& Stratta, G. 2007, GRB Coordinates Network, 7080, 1

Osborne, J. P., Beardmore, A. P., Evans, P. A., \& Goad, M. R. 2008, GRB Coordinates Network, 7687, 1

Paciesas, W. S., Meegan, C. A., Pendleton, G. N., et al. 1999, ApJS, 122, 465

Pei, Y. C. 1992, ApJ, 395, 130

Perri, M., Stratta, G., Barthelmy, S. D., et al. 2007, GRB Coordinates Network, 7059, 1

Piran, T. 1999, Phys. Rep., 314, 575

Piro, L., De Pasquale, M., Soffitta, P., et al. 2005, ApJ, 623, 314

Rees, M. J., \& Meszaros, P. 1998, ApJ, 496, L1

Romano, P., Campana, S., Chincarini, G., et al. 2006, A\&A, 456, 917

Sahu, D. K., Anupama, G. C., \& Misra, K. 2008, GRB Coordinates Network, 7700,1

Sari, R., Piran, T., \& Narayan, R. 1998, ApJ, 497, L17

Sbarufatti, B., Mangano, V., La Parola, V., \& Baumgartner, W. H. 2008, GRB Coordinates Network, 7694, 1

Schady, P., Mason, K. O., Page, M. J., et al. 2007, MNRAS, 377, 273

Schlegel, D. J., Finkbeiner, D. P., \& Davis, M. 1998, ApJ, 500, 525

Soderberg, A. M., Kulkarni, S. R., Fox, D. B., et al. 2005, ApJ, 627, 877

Sposetti, S. 2007, GRB Coordinates Network, 7090, 1

Stanek, K. Z., Matheson, T., Garnavich, P. M., et al. 2003, ApJ, 591, L17

Stanek, K. Z., Dai, X., Prieto, J. L., et al. 2007, ApJ, 654, L21

Starling, R. L. C., Wijers, R. A. M. J., Wiersema, K., et al. 2007, ApJ, 661, 787

Stratta, G., Fiore, F., Antonelli, L. A., Piro, L., \& De Pasquale, M. 2004, ApJ, 608,846

Stratta, G., Perri, M., \& Burrows, D. N. 2007, GRB Coordinates Network, 7079,

Uemura, M., Sasada, M., Arai, A., \& Uehara, T. 2007a, GRB Coordinates Network, 7062, 1

Uemura, M., Sasada, M., Arai, A., \& Uehara, T. 2007b, GRB Coordinates Network, 7094, 1

Updike, A. C., Hartmann, D. H., \& Rumstay, K. S. 2007, GRB Coordinates Network, 7084, 1

van der Horst, A. J., \& Wijers, R. A. M. J. 2007, GRB Coordinates Network, 7092,1

van Paradijs, J., Groot, P. J., Galama, T., et al. 1997, Nature, 386, 686

Vaughan, S., Goad, M. R., Beardmore, A. P., et al. 2006, ApJ, 638, 920

Wang, X., \& Loeb, A. 2000, ApJ, 535, 788

Watanabe, M., Nakaya, H., Yamamuro, Y., et al. 2005, PASP, 117, 870

Watson, D., Hjorth, J., Fynbo, J. P. U., et al. 2007, ApJ, 660, L101

Weingartner, J. C., \& Draine, B. T. 2000, in BAAS, 32, 1466

Yamazaki, R., Ioka, K., \& Nakamura, T. 2002, ApJ, 571, L31

Yamazaki, R., Ioka, K., \& Nakamura, T. 2003, ApJ, 593, 941

Yamazaki, R., Ioka, K., \& Nakamura, T. 2004, ApJ, 606, L33

Yamazaki, R., Toma, K., Ioka, K., \& Nakamura, T. 2006, MNRAS, 369, 311

Yoshida, M., Yanagisawa, K., Shimizu, Y., Toda, H., \& Kawai, N. 2007, GRB Coordinates Network, 7091, 1

Yuan, F., Rykoff, E. S., Rujopakarn, W., \& Swan, H. 2007, GRB Coordinates Network, 7061, 1

Zhang, B., \& Mészáros, P. 2004, Int. J. Mod. Phys. A, 19, 2385

Zhang, B., Fan, Y. Z., Dyks, J., et al. 2006a, ApJ, 642, 354

Zhang, B., Fan, Y. Z., Dyks, J., et al. 2006b, ApJ, 642, 354 